\title{
Some Biologically Important Aspects of Adsorption of Antibody on to Tissue Cells
}

\author{
BY J. H. HUMPHREY \\ National Institute for Medical Research, Mill Hill, London, N.W. 7
}

The reaction between antibody against a constituent of the cell surface and the corresponding antigen will usually damage the cell, especially if complement is present. Although the mechanism of such damage is obscure, so far as this Discussion is concerned there is no problem as to how the interaction comes about. It is less easy to see, however, how antibody against a completely extraneous antigen should so be able to 'sensitize' tissues that subsequent interaction with the antigen results in damage to at least some cells, and so produces the familiar features of anaphylaxis.

Most studies on anaphylaxis have been carried out on guinea pigs, using either active sensitization (with antigen), or passive sensitization with rabbit antibody. For quantitative studies passive sensitization is essential, and rabbit antibody is a fortunate choice since almost all rabbit antibody sensitizes guinea-pig tissues (a property which is not shared by all antibodies from other species, as is discussed below). The nature of the cells that become sensitized in such a way as to manifest obvious damage on subsequent contact with antigen has not been exactly determined. Mast cells are certainly among them (Mota, 1959; Humphrey \& Mota, $1959 a$ ); indeed, inasmuch as such cells are present in the connective tissue of most organs, and when damaged release powerful pharmacologically active substances, such as histamine and serotonin that act on smooth muscle and vascular endothelium, they may be the most important cells. However, mast cells constitute quite a small proportion of the cells in any tissue (less than $1 \%$ ), and there is at present no reason to suppose that antibodies sensitize mast cells exclusively. Anaphylaxis is accompanied by the release of other materials besides histamine and serotonin, such as 'slow reacting substance' (SRS-A) and bradykinin, and the origin of these last two is unknown (see review by Humphrey, 1962).

A characteristic feature of passive sensitization, either of the living guinea pig or of isolated tissues (e.g. lung, ileum, uterus) in vitro, is that the extent of sensitization, as judged by the severity of the manifestations on subsequent contact with antigen, increases with the duration of contact with the antibody. Although preformed antigen-antibody complexes per se are able to elicit at least some of the features of anaphylaxis, such as histamine release or smooth muscle contraction, they are very inefficient at doing so compared with antibody that has become 'fixed' on the tissues. Fixed antibody, on the other hand, is very efficient. Brocklehurst and I, using ${ }^{131}$ I-labelled antibody administered intravenously to a guinea pig $48 \mathrm{hr}$. previously, found that the presence of as little as $20 \mathrm{~m} \mu \mathrm{g}$. of antibody per g. of wet tissue was sufficient to sensitize the ileum so that it responded to contact with antigen in an organ bath by giving a maximal contraction. Since such small amounts are involved, lying below the limits for detection by many tests for antibody, it is not 
surprising that it was long thought that fixed antibody could exist in the absence of any antibody in the blood.

By studying the sensitization in vitro, by labelled antibody, of tissues whose response to antigen can be quantitated, it is possible to relate fixation of antibody to sensitization. Various tissues have been used in this way: lung fragments, from which the histamine released on contact with the antigen can be measured; mesentery fragments in which mast cell disruption can be estimated; and pieces of ileum, whose contraction in response to an excess of antigen is limited by the amount of antibody fixed and can be compared with contractions due to known amounts of histamine. These systems are used as models only, and should not be taken as reliable guides to the complex events of anaphylaxis in the whole animal.

Sensitization does not necessarily involve active uptake of antibody into cells. For example it occurs under circumstances in which active uptake is precluded, when tissues are left in contact with antibody in the cold or in the presence of reversible metabolic inhibitors. Furthermore, sensitized tissues can be de-sensitized (i.e. the antibody can be neutralized without damaging the cell) by contact with the antigen under similar circumstances. Both findings indicate that sensitization involves adsorption of antibody at the cell surface. Studies by Brocklehurst, Humphrey \& Perry (1961) bore this out, inasmuch as uptake by lung fragments of purified ${ }^{131}$ I-labelled rabbit gamma globulin (containing a high proportion of specific antibody) obeyed a typical adsorption isotherm. The tissue was maximally sensitized, however, when only a small fraction of the antibody ultimately adsorbed had been taken up. When such sensitized tissues were subjected to prolonged washing in Tyrode's solution, antibody was eluted, but never completely enough for the tissues to lose their sensitization. It might be expected that guinea-pig tissues would not be able to distinguish antibody from non-specific gamma globulin, and that antibody molecules would only be adsorbed in competition with similar nonantibody molecules. This proved to be the case, and the rate of uptake of antibody and extent of sensitization of the chopped lung were decreased by adding nonspecific gamma globulin (but not other plasma fractions). Binaghi, Liacopoulos, Halpern \& Liacopoulos-Briot (1962) have studied this aspect carefully, using a standardized technique for sensitizing pieces of ileum. They found that the gamma globulins of various species competed with rabbit antibody for sensitizing the ileum, but with decreasing effectiveness in the order rabbit, man, dog, guinea pig, rat, horse, cattle, pig, chick, goat. They also showed that the rate of sensitization varied little over the pH range $\mathbf{5 \cdot 8 - 8 \cdot 2}$ (Halpern et al. 1959), but was increased in isotonic media of low ionic strength (Binaghi et al. 1961), or in the presence of $0.8 \mathrm{M}$ urea (Binaghi et al. 1959).

So far the story seems reasonably straightforward, except in so far as the important adsorption sites are not known and that there is quite strong circumstantial evidence that antibody which has been in contact with tissue for a longer time is not only more extensively but also more firmly adsorbed than that which has been in contact for a shorter time. This argues that adsorption sites are heterogeneous, and has been interpreted by Mongar \& Schild (1962) as also implying a multi-point attachment. The story becomes complicated however when the capacity of different sera to sensitize guinea-pig tissues is compared. Differences are found both between and within species. For example, goat, chick, pig, cattle, horse and rat antibodies 
nearly always fail to sensitize guinea-pig tissues for anaphylaxis (Humphrey $\&$ Mota, $1959 \mathrm{~b}$ ). Inasmuch as these are the kinds of gamma globulin which competed least well with rabbit antibody, it may be that they are not adsorbed in the right way or at the right sites. Nevertheless, the few studies which have been made on the gross uptake of such globulins on to guinea-pig tissues failed to reveal any obvious distinction between them and rabbit antibody.

Furthermore, even when some antibodies of a species (rabbit, man or even the guinea pig itself) sensitize well, others may not do so at all. For example, human macroglobulin ( $\gamma 1 \mathrm{M}$ or $\beta 2 \mathrm{M}$ ) antibodies, and human reaginic (skin-sensitizing) antibodies, which may belong to the class of $\beta \mathbf{2 A}(\gamma \mathbf{1} \mathbf{A})$ globulins, do not sensitize guinea-pig tissues. The capacity of human globulins of these kinds to become 'fixed' on to guinea-pig skin cells has been studied by Ovary, Fudenberg \& Kunkel (1960) using the technique of direct and reversed passive cutaneous anaphylactic reactions. Such reactions depend upon sensitization of a localized area of the skin of the animal by intracutaneously injected antibody, or a suitably purified globulin fraction; and are elicited by subsequent intravenous injection of antigen, or of rabbit antibody against the globulin, in the direct or reversed reactions respectively. The animals also contain a suitable dye in their circulation, local extravasation of which reveals the presence and extent of any increased capillary permeability. Ovary \& Karush (1961) showed that sensitization depended upon the presence in the rabbit antibody molecule of a part with certain structural peculiarities. Porter (1959) first showed that the splitting of a small number of peptide bonds and reduction of some of the S-S bonds in rabbit gamma globulin caused the molecule to fall apart into three approximately equal parts, two of which (fragments I and II) contained the antibody combining sites and were antigenically very similar, while the third (fragment III) was antigenically distinct and was unrelated to antibody activity. Human gamma globulin is similarly split into two 'slow' (S) and one 'fast' (F) fragments (see review by Fahey (1962)). The presence of rabbit fragment III or human fragment $\mathrm{F}$ are associated with the ability to fix complement; they are also essential for the antibody or non-specific globulin to become fixed on and to sensitize guinea-pig tissues. Inasmuch as human $\beta \mathbf{2 A}(\gamma \mathbf{1 A})$ and $\beta \mathbf{2} \mathbf{M}(\gamma \mathbf{1} \mathbf{M})$ globulins do not contain the same $\mathbf{F}$ fragment as the other gamma globulins their failure to sensitize guinea-pig tissues is at least partly explained. However much remains to be discovered. For example, guinea-pig antibodies formed early in immunization have been found to be unable to sensitize guinea-pig tissues, while those formed later do so very well (Ovary \& Benacerraf 1962; White, Jenkins \& Wilkinson 1963). These antibodies differ in electrophoretic mobility, but both have similar molecular weights and both fix complement.

In other species the situation is at least as complex and much less thoroughly explored. Thus it has long been known that most human antibodies do not sensitize humar tissues in the same way as they would guinea-pig tissues. Those antibodies which do sensitize human tissues (reagins) are characteristically more labile to heat, and have a greater electrophoretic mobility than most other human antibodies, although their molecular weight and their general immuno-chemical nature is still a matter for dispute. In rats there is strong evidence that tissue-sensitizing antibodies are in some way different from others; they tend to be formed early in immunization, and especially when Bordetella pertussis has been used as an adjuvant 
(Mota 1962; Humphrey, Austen \& Rapp, 1963). Although the picture outlined above of a reversible adsorption of antibody onto cells as underlying sensitization may in principle be correct, there are certainly other, at present unknown, factors involved.

\section{REFERENCES}

Binaghi, R., Halpern, B. N. , Liacopoulos, P. \& Neveu, T. (1959). Promoting effect of urea on the in vivo passive sensitization of isolated plain muscles with precipitating antibody. Nature, Lond. 184, 1805.

Binaghi, R., Liacopoulos, P., Halpern, B. N., Lracopoulos-Briot, M. \& Block, C. (1961). Influence of the ionic strength of the medium on the velocity of passive anaphylactic sensitization in vitro. J. Immunol. 87, 269.

Binaghi, R., Liacopoulos, P., Halpern, B. N. \& Lracopoulos-Briot, M. (1962). Interference of non-specific gamma globulins with passive in vitro anaphylactic sensitization of isolated guinea pig intestine. Immunology, 5, 204.

Brockiehurst, W. E., Humphrey, J. H. \& Perry, W. L. M. (1961). The in vitro uptake of rabbit antibody by chopped guinea-pig lung and its relationship to anaphylactic sensitization. Immunology, 4, 67.

FAHEy, J. L. (1962). Heterogeneity of $\gamma$-Globulins. In Advances in Immunology, vol. 2. Academic Press.

Halpern, B. N., Liacopoulos, P., Liacopoulos-Briot, M., Binaghi, R., van Neer, F. (1959). Patterns of in vitro sensitization of isolated smooth muscle tissues with precipitating antibody. Immunology, 2, 351.

Humphrey, J. H. (1962). Biochemical mediators of antigen and antibody reactions. 4t Int. Congr. Allergology.

Humphrey, J. H., Austen, K. F. \& RAPP, H. J. (1963). In vitro studies of reversed anaphylaxis with rat cells. Immunology, 6 , in press.

Humphrey, J. H. \& Mota, I. (1959 $a)$. The mechanism of anaphylaxis: specificity of antigen-induced mast cell damage in anaphylaxis in the guinea pig. Immunology, $2,31$.

Humphrey, J. H. \& MotA, I. (1959b). The mechanism of anaphylaxis: observations on the failure of antibodies from certain species to sensitize guinea pigs in direct and reversed passive anaphylaxis. Immunology, 2, 19.

Mongar, J. L. \& Schild, H. O. (1962). Cellular mechanisms in anaphylaxis. Physiol. Rev. $2,226$.

MoтA, I. (1959). Effect of antigen and octylamine on mast cells and histamine content of sensitized guinea-pig tissues. $J$. Physiol. 147, 425.

MoтA, I. (1962). Failure of rat and rabbit antiserum to passively sensitize normal and pertussis-treated rats and mice so as to induce mast cell damage and histamine release on later contact with antigen. Immunology, 5, 11.

Ovary, Z. \& Benacerraf, B. (1962). Separation of skin sensitizing from blocking 7S antibodies in guinea-pig sera. Fed. Proc. 21, 21.

Ovary, Z., Fudenberg, H. \& Kunkel, H. G. (1960). Anaphylactic reactions in the skin of the guinea pig with high and low molecular weight antibodies and gamma globulins. J. exp. Med. 112, 953.

Ovary, Z., Karush, F. (1961). Studies on the immunologic mechanism of anaphylaxis. II. Sensitizing and combining capacity in vivo of fractions separated from papain digests of antihapten antibody. J. Immunol. 86, 146.

Porter, R. R. (1959). The hydrolysis of rabbit $\gamma$ globulin and antibody with crystalline papain. Biochem. J. 73, 119.

White, R. G., Jenkins, G. C. \& Wilkinson, P. C. (1963). 'The influence of components of Mycobacterium tuberculosis on the production of skin sensitizing and mast cell-reactive antibody in the guinea pig. Int. Arch. Allergy, N.Y., (in the press). 